\title{
Idiopathic Trigeminal Neuralgia Misdiagnosed as Secondary Trigeminal Neuralgia Caused by Neurogenic Tumor in the Infratemporal Fossa: A Case Report
}

\begin{abstract}
Jun-yong Cha ${ }^{1}$, Byung-chul Son ${ }^{1,2}$
${ }^{1}$ Department of Neurosurgery, Seoul St. Mary's Hospital, College of Medicine, The Catholic University of Korea, Seoul, ${ }^{2}$ Catholic Neuroscience Institute, College of Medicine, The Catholic University of Korea, Seoul, Republic of Korea

Corresponding author: Byung-chul Son Department of Neurosurgery, Seoul St. Mary's Hospital, Catholic Neuroscience Institute, College of Medicine, The Catholic University of Korea, 222 Banpo-daero, Seocho-gu, Seoul 06591, Republic of Korea

Tel: +82-2-2258-6122

Fax: $+82-2-594-4248$

E-mail: sbc@catholic.ac.kr

Received: June 23, 2020

A 64-year-old male patient presented with a 7-year history of chronic paroxysmal, stabbing, and electrical shock-like pain involving his mandibular nerve. After 1 year of successful medical treatment with carbamazepine, the pain was not controlled. Magnetic resonance imaging (MRI) revealed a small mass around the peripheral course of the right trigeminal nerve in the infratemporal fossa. The patient's trigeminal pain was very similar to that of classical trigeminal neuralgia. However, radiosurgery was performed to manage the infratemporal mass around the right trigeminal nerve. Despite the radiation therapy, pain worsened and became unbearably in the next 6 months. A suboccipital approach was performed for microvascular decompression of the neurovascular conflict, or an intradural sensory rhizotomy to block the transmission of the tumor pain. Typical neurovascular compression by the superior cerebellar artery was confirmed and only microvascular decompression was performed. The trigeminal pain that persisted for 7 years was resolved after the surgery. Even if a mass lesion located close to the peripheral course of the trigeminal nerve, possibility of classical trigeminal neuralgia due to neurovascular compression should be suspected if there is no direct involvement of the trigeminal nerve by the mass and the nature of trigeminal pain is consistent with classical trigeminal neuralgia.
\end{abstract}

Revised: August 1, 2020

Accepted: August 31, 2020
Key Words: Cancer pain; Microvascular decompression surgery; Pain; Trigeminal neuralgia

\section{INTRODUCTION}

Trigeminal neuralgia is characterized by recurrent and unilateral, brief electric shock-like pain triggered by innocuous stimuli, and is abrupt in onset and termination, but limited to the distribution of one or more divisions of the trigeminal nerve $^{1)}$. The diagnosis of trigeminal neuralgia is usually not difficult due to its characteristic symptoms. In the case of trigeminal neuralgia associated with facial pain, imaging study is essential to confirm whether the pain is due to neurovascular compression or space-occupying lesions (SOLs) in the trigeminal nerve pathway. If an SOL in the trigeminal nerve pathway is the cause of pain, it is called secondary trigeminal neuralgia.

Treatment of tumor-associated facial pain is not simple ${ }^{5)}$. Treatment modalities including chemotherapy, radiosurgery, and surgical resection, which are designated to treat the underlying tumor, have been associated with both elimination and initiation of facial pain in patients without pain history ${ }^{5)}$. We report a case that was mistaken for secondary trigeminal neu- ralgia for many years, due to the presence of asymptomatic SOL along the distal course of the trigeminal nerve in the infratemporal fossa on magnetic resonance imaging (MRI), despite the typical manifestations of classical trigeminal neuralgia caused by neurovascular compression.

\section{CASE REPORT}

A 64-year-old male patient presented with a 7-year history of chronic lancinating pain in his right jaw area (Fig. 1A). Seven year ago, a touch of his right chin elicited a sudden onset of brief stabbing pain without any special cause. The pain occurred 3 to 4 times a month. The patient was treated by a dentist without any improvement even after extraction of the lower molar. The intermittent pain in his jaw area lasted 3 to 4 years. The pain worsened 3 years prior to the presentation. Episodes of paroxysmal stabbing pain continued to occur when the patient's chin moved during speech, eating, facial washing, and brushing of teeth. The pain was so severe that 
his daily life became difficult (5-7/10 on Numerical Rating Scale [NRS]-11). The patient was treated in a pain clinic and was diagnosed with trigeminal neuralgia. His pain improved with medication (carbamazepine $400 \mathrm{mg} /$ day), but after a year the pain was refractory to the medication.

MRI was performed to evaluate the refractory neuralgic pain, which revealed a neurogenic tumor in the right infratemporal area (Fig. 1). Due to the location of the mass in the infratemporal fossa close to the peripheral branch of the trigeminal nerve, the patient was referred to a neurosurgeon in a tertiary hospital, for observation of the interval changes of the mass and drug treatment. For the next 2 years, the pain persisted despite treatment, but the patient endured the pain because the tumor was difficult to operate.

The pain intensified again a year before the visit. Paroxysmal stabbing pain occurred even minor facial movement. The intense pain interfered with eating and facial washing for 6 months. The patient was recommended radiosurgery for which. He visited our outpatient clinic for a second opinion or an alternative option.

On physical examination, no objective sign of sensory deficit in the distribution of the right trigeminal nerve or weakness of masseter muscle was found. No allodynia or paresthesia in the mandibular division of the trigeminal nerve was detected.
Examination of the other cranial nerves were normal and revealed no dental problems. Pain symptoms were pathognomonic of typical, primary trigeminal neuralgia. A trigger point for evoked trigeminal pain was identified in the right gingiva. Considering the refractory condition and radiological findings of the lesion suggesting a neurotic tumor, a consultation with a head and neck surgeon was requested for mass excision (Fig. 2A). However, the head and neck surgeons recommended observation with pain control because the tumor was small and not a life-threatening lesion. Carbamazepine $(600 \mathrm{mg} /$ day) and gabapentin $(300 \mathrm{mg} /$ day) were initiated. During the followup visit, the patient still complained of pain and thus, oxcarbazepine (300 mg/day) and pregabalin (150 mg/day) were added. We thought that his trigeminal pain might be a typical primary trigeminal neuralgia due to neurovascular conflict (Fig. 1D). However, radiation treatment was recommended due to a possible compression of the peripheral trigeminal nerve by the infratemporal mass.

Radiosurgery of the infratemporal mass was conducted using 18 Gy at $80 \%$ isodose curve (maximum 22.5 Gy) (Fig. 2B). No acute radiation-related complications developed following radiosurgical treatment. After radiation therapy, the pain aggravated for a month (7/10, NRS-11), which led to a prescription of steroid (prednisolone $20 \mathrm{mg}$ /day) for 2 months with a com-
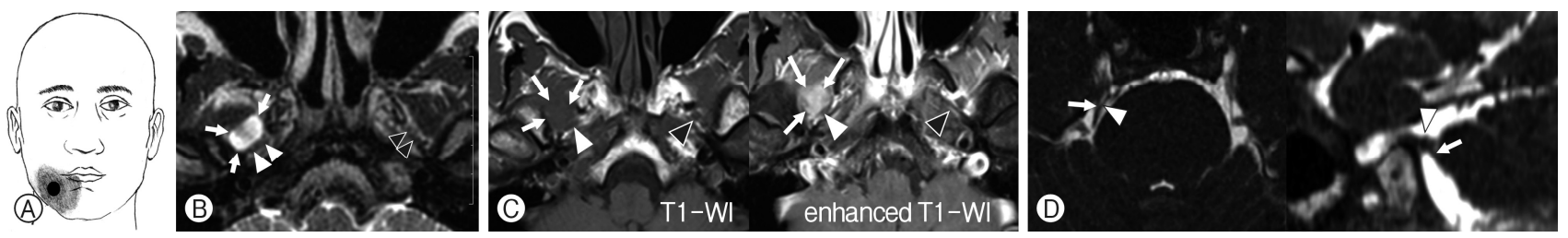

Fig. 1. Magnetic resonance imaging showing the distribution of facial pain and the mass in the right infratemporal fossa. (A) Distribution of facial pain. Black circle denotes the trigger point of facial pain. (B) Axial T2-weighted image revealed about $1.3 \mathrm{~cm}$ cystic lesion (arrows) in the right infratemporal fossa along the course of the trigeminal nerve (TN). The right mandibular nerve (white arrowheads) is located at the margin of the lesion. Note the location of left mandibular nerve (left arrowheads) in the infratemporal fossa. (C) Tl-weighted (left) and enhanced T1-weighted (right) images of the lesion in the right infratemporal fossa. The lesion is poorly enhanced. The white arrowheads indicate the right mandibular nerve and the black arrowhead represents the left mandibular nerve. (D) Axial (left) and oblique sagittal (right) 3-dimensional fast imaging employing steady-state acquisition (FIESTA) images showing neurovascular insults in the right TN. The right superior cerebellar artery (arrowhead) is in contact with the right TN (arrow).
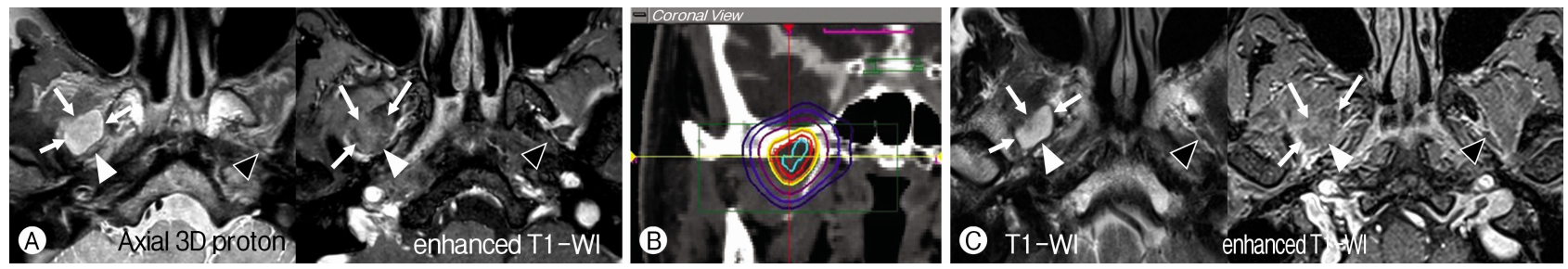

Fig. 2. Follow-up magnetic resonance imaging (MRI) and radiation treatment. (A) Axial 3-dimensional proton density image (left) and enhanced T1-weighted (right) images of the mass lesion. Compared with the MRI performed 3 years ago, it showed no discernible interval changes. (B) Dosimetry planning for radiosurgery. A dose of $18 \mathrm{~Gy}$ was given to the mass at $80 \%$ isodose curve (maximum dose, $22.5 \mathrm{~Gy}$ ). (C) T1-weighted (left) and enhanced T1-weighted (right) images obtained at 8 months post-radiation treatment. The lesion is slightly enlarged with slight enhancement for the radiation treatment. The white arrowheads indicate the right mandibular nerve and the black arrowhead represents the left mandibular nerve. 


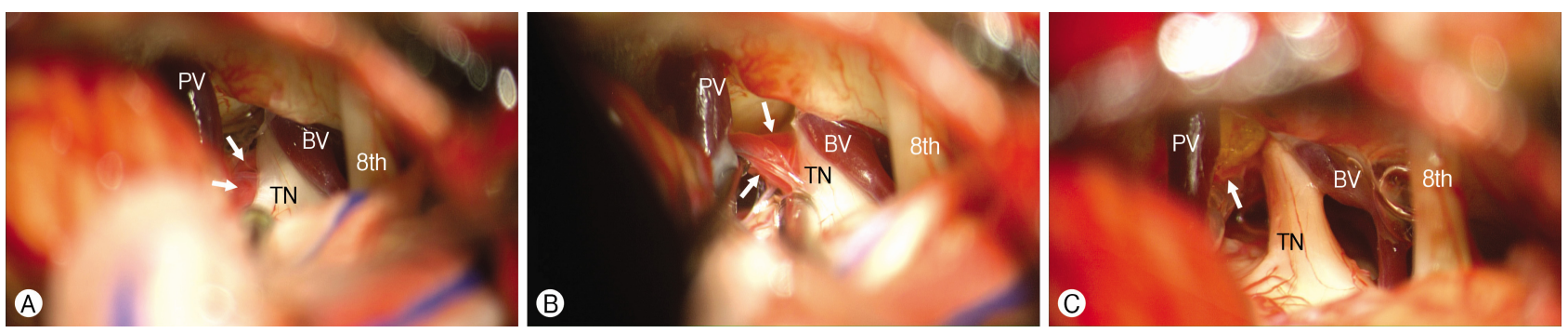

Fig. 3. Intraoperative findings during microvascular decompression. (A) The right trigeminal nerve (TN) is deviated by the arachnoid and compressed by the 2 loops of the superior cerebellar artery (arrows). (B) The loops of the superior cerebellar artery (arrows) are dissected from the TN. (C) After transposition of the superior cerebellar artery (arrow) to the tentorium and dissection of the bridging vein and surrounding arachnoid, the TN is free of the vascular compression. BV: bridging vein along the course of the TN root; PV: superior petrosal vein; 8th: the vestibulocochlear nerve.

bination of carbamazepine ( $600 \mathrm{mg} /$ day), oxcarbazepine ( 450 $\mathrm{mg} /$ day $)$, pregabalin $(225 \mathrm{mg} /$ day $)$ and amitriptyline $(10 \mathrm{mg} /$ day). Treatment with this combination regimen resulted in transient improvement (NRS-5-6/10). Additional treatment included carbamazepine $600 \mathrm{mg} /$ day), oxcarbazepine $(300 \mathrm{mg} /$ day), and pregabalin $(150 \mathrm{mg} /$ day $)$. Even after 6 months of radiation therapy, the pain did not improve and continued. Eight months later, severe and persistent pain prevented the intake of water and food. MRI was performed to evaluate the need for additional treatment to control the pain. Compared with the MR image before the radiosurgery, the new MRI did not reveal any change in tumor size or signal intensity (Fig. 2C). Neuroablative treatments to block the pain transmission using radiofrequency or intradural trigeminal rhizotomies were considered as curative surgery was contraindicated. However, the patient's pain was similar to that of typical trigeminal neuralgia and MRI showed a suspicion of neurovascular conflict involving the right trigeminal nerve, and therefore, the possibility of primary trigeminal neuralgia in a patient with a coincidence of infratemporal mass lesion was not excluded. We selected an intradural exploration because if the surgical findings revealed a pattern of trigeminal neuralgia in which the nerve was severely compressed by the offending vessels, only a decompression of the trigeminal nerve was performed, and if the neurovascular compression was not significant, an intradural trigeminal sensory rhizotomy was the alternative. After explaining the surgical plan to the patient and the guardian, obtaining informed consent, an intradural exploration was performed via suboccipital approach.

In the surgical findings showed arachnoid tethering and severe compression of the right trigeminal nerve ventrally by the loops of the superior cerebellar artery (SCA) (Fig. 3A). The SCA was carefully separated from the trigeminal nerve and transposed using glue on the tentorial dura (Fig. 3B). A tributary of the transverse pontine vein attached to the dorsal caudal side of the trigeminal nerve was carefully dissected and glued to the tentorial surface (Fig. 3C). Neurovascular compression by SCA, which caused severe deformation and compression of the trigeminal nerve, was considered typical of classical trigeminal neuralgia, and therefore, no partial sensory rhizo- tomy (PSR) was performed. After recovery from anesthesia, the chronic and severe pain in the V3 region disappeared. Neurological examination revealed mild paresthesia in the V3 region and no abnormalities in other cranial nerve functions. Postoperative recovery was uneventful. One month after surgery, the patient discontinued with carbamazepine and gabapentin and in 2 months, all drugs, including anticonvulsants, were stopped. Until one year after surgery, the pain of trigeminal neuralgia did not recur, and his daily life was not affected. In the MRI examination performed 1 year after surgery, the mass of the right infratemporal fossa showed no growth compared to the preoperative image.

\section{DISCUSSION}

\section{Classical and Secondary Trigeminal Neuralgia and Painful Trigeminal Neuropathy}

Trigeminal neuralgia is characterized by recurrent and unilateral, brief electric shock-like pain, abrupt in onset and termination, limited to the distribution of one or more divisions of the trigeminal nerve, and triggered by innocuous stimuli ${ }^{1)}$. It may be idiopathic or resulting from another diagnosed disorders. Additionally, there may be concomitant continuous pain of moderate-intensity within the distribution of the affected nerve branch $^{1)}$. The current classification of trigeminal neuralgia is based on the etiology of headache and facial pain: classic, secondary, and idiopathic. Classical trigeminal neuralgia is characterized by vascular compression of the respective nerve based on imaging or surgery. Strictly speaking, classical trigeminal neuralgia is secondary to neurovascular compression, but it is advantageous to separate it from other causes for treatment $^{1)}$.

Secondary trigeminal neuralgia is caused by underlying disease or conditions contributing to neuralgia, and established etiological factors include tumors in the cerebellopontine angle, arteriovenous malformation, and multiple sclerosis ${ }^{1)}$. MRI is the best modality available to identify the underlying causes 
Table 1. Diagnostic criteria of trigeminal neuralgia

\begin{tabular}{ll}
\hline \hline A Recurrent paroxysms of unilateral facial pain in the distribution(s) of one or more divisions of the trigeminal nerve, with no \\
radiation beyond, and fulfilling criteria B and C. \\
B Pain has all of the following characteristics: \\
1 Lasting from a fraction of a second to 2 minutes \\
2 Severe intensity \\
3 Electric shock-like, shooting, stabling or sharp in quality \\
C Precipitated by innocuous stimuli within the affected trigeminal distribution \\
D Not explained better by another ICHD-3 diagnosis
\end{tabular}

ICHD-3: International Classification of Headache Disorders, $3^{\text {rd }}$ edition.

[Reprinted from "Headache Classification Committee of the International Headache Society (IHS) The International Classification of Headache Disorders, 3rd edition.", by Headache Classification Committee International Headache Society, 2018, Cephalalgia, 38, pp. 1-211. Copyright 2018 by the Sage. Reprinted with permission].

of secondary trigeminal neuralgia ${ }^{1)}$. Idiopathic trigeminal neuralgia refers to trigeminal neuralgia without significant abnormalities based on electrophysiological tests or $\mathrm{MRI}^{1)}$. The common diagnostic criteria associated with the 3 types of trigeminal neuralgia are listed, in addition to recurrent paroxysm of unilateral facial pain in the distribution of one or more divisions of the trigeminal nerve (Table 1 ).

\section{Difficulty in Surgical Decision Making for the Surgical Treatment of Secondary Trigeminal Neuralgia}

In the current case, the facial pain was initially interpreted as a symptom associated with typical trigeminal neuralgia and medical treatment with carbamazepine was effective for a year. However, the patient was diagnosed with secondary trigeminal neuralgia after the MRI revealed a mass in the infratemporal fossa in the vicinity of the peripheral trigeminal nerve. The patient was transferred to another hospital. Although he was diagnosed with secondary trigeminal neuralgia from the mass lesion, for unknown reasons, the patient continued treatment with ineffective drugs. Indeed, the patient's pain showed characteristics of typical trigeminal neuralgia, and the apparent trigger point causing pain by innocuous stimuli was also confirmed, and thus fully met the diagnostic criteria of classic trigeminal neuralgia. No sensory abnormalities in the affected division of the trigeminal nerve were detected. The pain of classic trigeminal neuralgia is known to increase in severity with time ${ }^{1)}$. Despite the presence of a small mass in the peripheral course of the trigeminal nerve, because the patient's symptoms are very similar to those of the classic trigeminal neuralgia, a possibility of neurovascular conflict should be suspected.

Another difficulty complicating the diagnosis of classic trigeminal neuralgia in this case was that MRI revealed neurovascular contact with the right trigeminal nerve; however, it was not considered as a serious neurovascular compression ${ }^{3,4)}$. Although neurovascular contact with the trigeminal nerve is considered as a frequent cause of classical trigeminal neuralgia $^{3)}$, a neurovascular contact on both the symptomatic and asymptomatic sides is common ${ }^{3)}$. MRI or surgical findings of neurovascular compression associated with morphological changes (atrophy and/or displacement of the trigeminal nerve root) are needed to diagnose classical trigeminal neuralgia caused by neurovascular compression ${ }^{1,3)}$. In the current case, neurovascular contact by a SCA and a small bridging vein was identified in the right trigeminal nerve (Fig. 3A). However, the neurovascular contact in MRI was not severe enough to be considered as nerve atrophy or displacement ${ }^{3}$. Finally, neurovascular compression by SCA, which showed nerve displacement in the surgical findings, was confirmed, but it was still difficult to diagnose classic trigeminal neuralgia based on preoperative MRI findings. A repeat MRI of the relationship between the infratemporal mass and the peripheral trigeminal nerve revealed the location of mass immediately next to the trigeminal neve; however, it was not a mass of the nerve itself and did not directly involve the nerve.

A suboccipital approach was performed to perform $\mathrm{PSR}^{2)}$ or microvascular decompression depending on the severity of neurovascular compression in the surgical field of view. Since severe neurovascular compression of the trigeminal nerve by the SCA and tethering by the arachnoid membranes were confirmed, the need for PSR was not felt. Transposition of the SCA to the tentorial dura mater and arachnolysis around the trigeminal nerve were performed successfully. The patient's severe trigeminal neuralgia, which lasted 7 years, was resolved immediately after surgery, without any recurrence. In hindsight, if we had considered the possibility of neurovascular compression rather the infratemporal mass as the factor triggering the pain, the patient's pain and suffering would have been reduced substantially.

\section{CONCLUSION}

Even in patients with secondary trigeminal neuralgia sus- 
pected due to a mass surrounding the peripheral trajectory of the trigeminal nerve in the imaging study, the possibility of classical trigeminal neuralgia caused by neurovascular compression should be considered depending on the consistent pain pattern.

\section{CONFLICTS OF INTEREST}

No potential conflict of interest relevant to this article was reported.

\section{REFERENCES}

1. Headache classification committee of the international headache society (IHS): The international classification of headache disorders, $3^{\text {rd }}$ edition. Cephalalgia 38:1-211, 2018

2. Koopman JS, de Vries LM, Dieleman JP, Huygen FJ, Stricker $\mathrm{BH}$, Sturkenboom MC: A nationwide study of three invasive treatments for trigeminal neuralgia. Pain 152:507-513, 2011

3. Maarbjerg S, Wolfram F, Gozalov A, Olesen J, Bendtsen L: Significance of neurovascular contact in classical trigeminal neuralgia. Brain 138:311-319, 2015

4. Miller JP, Acar F, Hamilton BE, Burchiel KJ: Radiographic evaluation of trigeminal neurovascular compression in patients with and without trigeminal neuralgia. J Neurosurg 110:627-632, 2009

5. Reddy GD, Wagner K, Phan J, DeMonte F, Raza SM: Management of skull base tumor-associated facial pain. Neurosurg Clin N Am 27:337-344, 2016 